\title{
An Experimental Assessment of the Effects of K-Pop Music Video Exposure on Teenagers' Negative Body Image
}

\author{
Abigail Lin $^{1 *}$, Denisa Vlasceanu ${ }^{2}$, Madalina Vlasceanu ${ }^{3}$ \\ ${ }^{1}$ Madison West High School, Madison, USA \\ ${ }^{2}$ Mihai Viteazul National College, Bucharest, Romania \\ ${ }^{3}$ Princeton University, Princeton, USA \\ Email: ^abigail.lin@gmail.com
}

How to cite this paper: Lin, A., Vlasceanu, D., \& Vlasceanu, M. (2021). An Experimental Assessment of the Effects of K-Pop Music Video Exposure on Teenagers' Negative Body Image. Open Journal of Social Sciences, 9, 40-46.

https://doi.org/10.4236/jss.2021.98004

Received: June 29, 2021

Accepted: July 31, 2021

Published: August 3, 2021

Copyright $\odot 2021$ by author(s) and Scientific Research Publishing Inc. This work is licensed under the Creative Commons Attribution International License (CC BY 4.0).

http://creativecommons.org/licenses/by/4.0/

\begin{abstract}
Exposure to K-Pop music videos has been associated with increased negative body image in prior correlational work. Here, we experimentally assessed the causal link between exposure to K-Pop music videos and negative body image in teenagers. To investigate, we recruited a sample of 76 teenagers from high schools in the United States and Europe. Participants first rated their body image perception (pre-test) after which they were randomly assigned to watch a K-Pop music video (Experimental Condition) or a classical music video (Control Condition). Finally, participants rated their body image again (posttest). We did not find a causal relationship between K-Pop music video exposure and negative body image in teenagers. In exploratory analyses, we found that baseline levels of negative body image are lower in European compared to American teenagers. We discuss these results in the context of beauty standards across cultures.
\end{abstract}

\section{Keywords}

Negative Body Image, K-Pop Music Videos, Beauty Standards

\section{Introduction}

Lee Ji-Eun, known as IU, is a teenage K-Pop singer who suffered from bulimia nervosa, an eating disorder characterized by binge eating followed by purging. She combined this with a strict diet of less than 400 calories a day, reportedly only eating one apple, two sweet potatoes, and one protein shake a day (Korea Herald, 2011). IU's story is neither unique nor exclusive to women-Korean 
boys were just as likely to internalize the ideal of beauty disseminated by the media (You \& Shin, 2020). With the advent of social media, such unhealthy behaviors are spreading at an accelerated rate, with younger populations being most vulnerable. In a recent study, South Korean kindergarten girls reported wanting to be thinner, a desire correlated with how long the girls had been exposed to K-Pop TV stations (Kim \& Han, 2020). However, prior research has not established whether there is a causal connection between K-Pop music video exposure and negative body image, as most prior studies have relied on correlational designs. Thus, in this investigation, we are interested in experimentally assessing whether such causal link exists, particularly in teenagers. Adolescents are a population of particular interest given their susceptibility to mental health issues (Farley, 2020) and vulnerability to negative influences by media-established beauty standards (Kinder \& Sears, 1985).

Moreover, research has also suggested that there are differences in how prone people of different backgrounds are to experience distress associated with negative body image (Kim \& Aubrey, 2015). Compared to people of Caucasian descent, people of Asian descent have been found to have lower self-esteem, place greater importance on appearance, and be more critical of their bodies (Jung \& Lee, 2006). Therefore, we are also interested in observing whether the negative body image perception, as influenced by K-Pop music video exposure differs across backgrounds.

To investigate, we designed a study in which participants rated their body image perception after which they were exposed to either a K-Pop music video or a classical music video. Lastly, participants rated their body image again. We hypothesized that participants who were exposed to the K-Pop music video would increase their negative body image perception more compared to participants who were exposed to the control, classical music video (H1). We also hypothesized this effect would be larger for Asian participants compared to Caucasian participants (H2). Finally, we hypothesized this effect would not be different for male compared to female participants (H3). We did not have hypotheses for how the effect would interact with participants' country of residence (i.e., United States versus Romania).

\section{Method}

\subsection{Open Science Practices}

The materials and data can be found on the OSF platform here: https://osf.io/nh6r9/.

The data analysis in Python can be found as a Jupyter Notebook here: https:/github.com/mvlasceanu/kpopexposure/blob/main/KPOP-Copyl.ipynb.

\subsection{Participants}

We used a convenience sample of 76 teenagers. Of them, 50 were high school students in the United States (Mage $=16.06$, SDage $=0.79,78.9 \%$ women $)$, and 
26 were high school students in Romania (Mage $=17.26$, SDage $=0.53,71 \%$ women). All participants passed pre-established attention checks.

\subsection{Stimulus Materials}

We used 3 music videos from Youtube. The videos in the experimental condition were of popular K-Pop groups. The criteria for choosing these videos were popularity (i.e., they have over 10 million views on YouTube) and the camera angles (i.e., they include full-body shots). We chose a female K-Pop group (i.e., ITZY) and a male K-Pop artist (i.e., Wonho) to match the video we exposed participants to with their self-reported gender, as prior work established that people are more influenced by others with whom they share common characteristics (Abrams, Wetherell, Cochrane, Hogg, \& Turner, 1990). The video we chose for the control condition has the same length as the experimental video (i.e., $3 \mathrm{mi}$ nutes) but features an orchestra of both male and females playing classical music.

\subsection{Design and Procedure}

Data collection occurred between October and December 2020. Participants were told they would participate in an experiment about music preferences in high schoolers and were directed to the survey on the Qualtrics platform. After completing the informed consent form, participants were asked to complete a series of demographic information and the body image questionnaire (pretest). Then they were randomly assigned to either the experimental or control condition. Participants in the experimental condition were exposed to the K-Pop music video that matched their self-reported gender. Participants in the control condition were exposed to the classical music video. Finally, in the third phase, participants were asked to complete the body image questionnaire again (posttest), after which they were debriefed.

\subsection{Measures}

To measure participants' body image, we used a body index questionnaire (e.g., “Do you feel you need to diet?") adapted from Kim \& Han (2020). In the demographic questionnaire we asked participants to report their age, gender, and country of residence.

\section{Results}

\subsection{Data Preprocessing}

We excluded outliers based on pre-established criteria (i.e., participants whose body image questionnaire score deviates from the mean score by more than three standard deviations). Only one participant's data was excluded based on this criterion. We conducted the statistical analyses on the rest of the participants $(\mathrm{N}=75)$.

\subsection{Main Results}

To test our first hypothesis, that participants exposed to a K-Pop music video 
would increase their negative body image more compared to participants exposed to a Classical music video, we compared the experimental group's change in the body image index (Mchange $=0.023$; SDchange $=0.31$ ) to the control group's (Mchange $=-0.11$; SDchange $=0.37$ ). The two groups' difference scores were not statistically significantly different in an independent sample t-test ( $p=$ $0.246, \mathrm{CI}=[-0.059,0.227], \mathrm{d}=0.245, \mathrm{t}(74)=1.165$ ) (Figure $1(\mathrm{~A})$ ). Thus, we did not find support for our first hypothesis, that exposure to K-Pop music videos increase teenagers' negative body image.

To test our second hypothesis, that the effect of K-Pop music video on teenagers' body image is stronger for Asian compared to Caucasian participants, we conducted a between-subjects ANOVA with Ethnicity (Caucasian/Asian) and Condition (Experimental/Control) as between subject independent variables and change in negative body image as the dependent variable we found a nonsignificant main effect of Ethnicity ( $p=0.202)$, a nonsignificant main effect of Condition $(p=0.257)$, and a nonsignificant interaction $(p=0.114)$. Therefore, we did not find any significant ethnicity differences in how K-Pop music videos influence teenagers' body image.

In exploratory analyses we investigated the effect of K-Pop music video on teenagers' body image as it interacts with participants' gender. In a betweensubjects ANOVA with Gender (Male/Female) and Condition (Experimental/ Control) as between subject independent variables and change in negative body image as the dependent variable we found a marginally significant main effect of gender $(p=0.068)$, a nonsignificant main effect of condition $(p=0.323)$, and nonsignificant interaction $(p=0.243)$ (Figure $1(B))$. Therefore, we did not find any significant gender differences in how K-Pop music videos influence teenagers' body image.

Additionally, we investigated the effect as it interacts with participants' country of residence. In a between-subjects ANOVA with Country (US/Romania) and Condition (Experimental/Control) as the between subject independent variables and change in negative body image as the dependent variable we found a nonsignificant main effect of country $(p=0.286)$, a nonsignificant main effect of condition $(p=0.247)$ and nonsignificant interaction $(p=0.415)$ (Figure $1(D))$. Therefore, we did not find any significant country of residence differences in how K-Pop music videos influence teenagers' body image.

Finally, in exploratory analyses we were interested in comparing the baseline negative body image of teenagers in the two countries we collected data from. In an independent sample t-test comparing baseline negative body image of participants in Romania with participants in the US we found a significant difference $(p=0.007, \mathrm{CI}=[0.154,1.387], \mathrm{d}=0.582, \mathrm{t}(74)=58.42$, suggesting that teenagers in Romania have a less negative body image at baseline (Figure 2).

\section{Discussion}

In an experimental study, we did not find evidence for the hypothesis that 
A

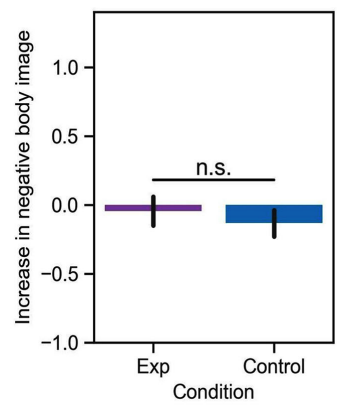

B

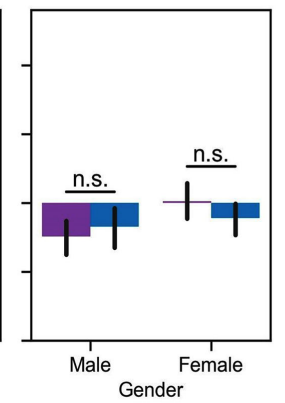

C

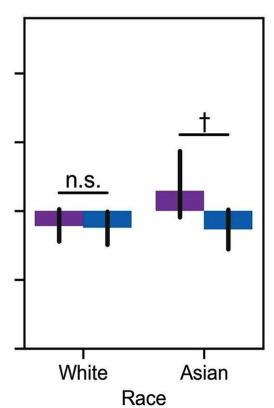

D

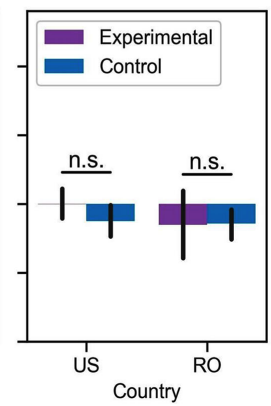

Figure 1. Increase in negative body image as a function of condition (panel (A)), gender (panel (B)), race (panel (C)), and Country (panel (D)). The experimental condition is depicted in purple, the control condition is depicted in blue. Error bars denote $95 \%$ confidence intervals around the means.



Figure 2. Negative body image at pretest (baseline) as a function of country. Participants in the United States depicted in red, participants in Romania depicted in yellow. Error bars denote $95 \%$ confidence intervals around the means.

exposure to K-Pop music videos causally impacts teenagers' body image. This result offers additional context to the correlational work reporting an association between K-Pop exposure and negative body image in kindergartner girls (Kim \& Han, 2020). Our study suggests that either another variable caused both the increased K-Pop exposure and the negative body image, or that the negative body image caused the increase in K-Pop music video consumption in Kim and Han's (2020) study. Alternatively, it could be that the single exposure to K-Pop music videos tested here was not sufficient to trigger a response. To understand the effects of repeated exposure to such content on teenagers' body image, a longitudinal experimental study is needed. Such investigation could also reveal the boundary conditions of a potential effect of K-Pop music video exposure on teenagers' body image, by uncovering the threshold, or minimal exposure necessary to elicit an effect.

In exploratory analyses, we found that American teenagers view their bodies more negatively compared to European teenagers. This finding is consistent with 
prior work showing that adolescents in the US are more likely than adolescents in European countries to engage in weight loss behaviors such as dieting ( $\mathrm{Za}$ borskis, Petronyte, Sumskas, Kuzman, \& Iannotti, 2008). This difference underlines the high emphasis on body image and ideal beauty standards reinforced in the United States from a young age. This field would greatly benefit from future research investigating both the consequences of standards of beauty US teenagers impose on themselves (e.g., in the context of eating disorders), and ways to mitigate potential negative effects.

To ensure the reliability of these results future work replicating our findings with a larger, more representative sample of adolescents would be necessary. Moreover, future work should investigate the connection between K-Pop music video exposure and teenagers' body image in additional countries, to explore the generalizability of the results we report here. Finally, future research investigating the effects of repeated exposure to K-Pop music videos on teenagers' body image would increase the ecological validity of the design implemented here.

\section{Acknowledgements}

We thank Polygence for funding this project, and reviewers for crucial suggestions.

\section{Conflicts of Interest}

The authors declare no conflicts of interest regarding the publication of this paper.

\section{References}

Abrams, D., Wetherell, M., Cochrane, S., Hogg, M. A., \& Turner, J. C. (1990). Knowing What to Think by Knowing Who You Are: Self-Categorization and the Nature of Norm Formation, Conformity and Group Polarization. British Journal of Social Psychology, 29, 97-119. https://doi.org/10.1111/j.2044-8309.1990.tb00892.x

Farley, H. R. (2020). Assessing Mental Health in Vulnerable Adolescents. Nursing, 50, 48-53. https://doi.org/10.1097/01.NURSE.0000697168.39814.93

Jung, J., \& Lee, S. H. (2006). Cross-Cultural Comparisons of Appearance Self-Schema, Body Image, Self-Esteem, and Dieting Behavior between Korean and US Women. Family and Consumer Sciences Research Journal, 34, 350-365. https://doi.org/10.1177/1077727X06286419

Kim, H., \& Han, T. I. (2020). Body Image Concerns among South Korean Kindergarteners and Relationships to Parental, Peer, and Media Influences. Early Childhood Education Journal, 49, 177-184. https://doi.org/10.1007/s10643-020-01059-z

Kim, K. B., \& Aubrey, J. S. (2015). A Cross-Cultural Comparison of Cognitive and Affective Mediators in the Relationship between Media Use and Body Image Disturbance: Focusing on US and Korean Women. Asian Journal of Communication, 25, 507-524. https://doi.org/10.1080/01292986.2014.995681

Kinder, D. R., \& Sears, D. O. (1985). Public Opinion and Political Action. In G. Lindzey, \& E. Aronson (Eds.), The Handbook of Social Psychology (3rd ed., pp. 659-741). Random House. 
The Korea Herald (2011).

http://biz.heraldcorp.com/common prog/newsprint.php?ud=20111228000809

You, S., \& Shin, K. (2020). Sociocultural Influences, Drive for Thinness, Drive for Muscularity, and Body Dissatisfaction among Korean Undergraduates. International Journal of Environmental Research and Public Health, 17, Article No. 5260. https://doi.org/10.3390/ijerph17145260

Zaborskis, A., Petronyte, G., Sumskas, L., Kuzman, M., \& Iannotti, R. J. (2008). Body Image and Weight Control among Adolescents in Lithuania, Croatia, and the United States in the Context of Global Obesity. Croatian Medical Journal, 49, 233-242.

https://doi.org/10.3325/cmj.2008.2.233 